\title{
Satisfacción laboral en docentes de la facultad de medicina de una universidad pública
}

\section{Job satisfaction in professors from a public university medical school}

\author{
Víctor Cruz Boullosa ${ }^{1, a}$, Ivonne Bernui Leo ${ }^{2, b}$ \\ ${ }^{1}$ Departamento Académico de Ciencias Morfológicas, Facultad de Medicina, Universidad Nacional Mayor de San Marcos. Lima, Perú. \\ ${ }^{2}$ Centro de Investigación de Bioquímica y Nutrición, Facultad de Medicina, Universidad Nacional Mayor de San Marcos. Lima, Perú. \\ a Profesor asociado de anatomía humana. \\ ${ }^{\mathrm{b}}$ Magister en nutrición, profesor principal de investigación. ORCID: https://orcid.org/0000-0001-5289-8084
}

\begin{abstract}
Correspondencia:
Víctor Cruz Boullosa

vcruzboullosa@gmail.com
\end{abstract}

Recibido: 10 de mayo 2019

Aceptado: 22 de agosto 2019

Publicación en línea: 28 de diciembre 2019

Conflictos de interés: Los autores declaran no tener conflictos de interés.

Fuente de financiamiento:

Autofinanciado

Citar como: Cruz V, Bernui I. Satisfacción laboral en docentes de la facultad de medicina de una universidad pública. An Fac med. 2019;80(4):494-7. DOI: https:/doi.org/10.15381/anales.v80i4.16151
An Fac med. 2019;80(4):494-7 / DOI: https://doi.org/10.15381/anales.v80i4.16151

\section{Resumen}

Introducción. La satisfacción laboral es importante para lograr un servicio de calidad en la organización. Objetivo. Determinar la satisfacción laboral en docentes nombrados del Departamento Académico de Ciencias Morfológicas de la Facultad de Medicina de la Universidad Nacional Mayor de San Marcos, en el año 2014. Métodos. Estudio transversal. Se aplicó el cuestionario del Índice Descriptivo del Trabajo (JDI) a 21 docentes nombrados, mediante el cual se determinó la satisfacción laboral general y cinco dimensiones especificas: trabajo actual, remuneración, oportunidades, colegas y supervisión. Resultados. Se entrevistaron 14 docentes de anatomía y 7 docentes de histología y embriología. Se encontró un $71 \%$ de varones, con una edad promedio 62,3 años, la mayoria con un tiempo de servicio entre 30 a 39 años (61\%). La dimensión con mayor puntaje promedio fue la satisfaccióncon el trabajo actual (35,1 puntos); mientras la dimensión con mayor insatisfacción es la remuneración (17,8 puntos). Conclusión. A pesar de la edad y tiempo de servicio, los docentes de morfología mostraron satisfacción por el trabajo actual, independiente de la remuneración y otras variables.

Palabras clave: Satisfacción en el Trabajo; Docentes; Facultades de Medicina; Estudios Transversales; Encuestas y Cuestionarios (fuente: DeCS BIREME).

\section{Abstract}

Introduction. Job satisfaction is important for the quality of service achievement in the organization. Objective. To determine job satisfaction in professors, who were working on Academic Department of Morphological Sciences of Facultad de Medicina in Universidad Nacional Mayor de San Marcos, during 2014. Methods. A transversal study. The Descriptive Work Index Questionnaire (JDI) was applied in 21 professors, to evaluate general job satisfaction and five specific dimensions: Current work, remuneration, opportunities, colleagues and supervision. Results. 14 professors of Anatomy and 7 professors of histology and embryology were interviewed. $71 \%$ of people were men, with an average age of 62,3 years, the majority with a service time between 30 to 39 years $(61 \%)$. The dimension with the highest average score was satisfaction with current work (35,1 points); while the dimension with greater dissatisfaction is the remuneration (17,8 points). Conclusion. Despite the age and time of service, morphology professors show satisfaction with current work, independently of remuneration and other variables.

Keywords: Job Satisfaction; Faculty; Schools, Medical; Cross-Sectional Studies; Surveys and Questionnaires (source: MeSH NLM). 


\section{INTRODUCCIÓN}

Cuando el comportamiento organizacional es estudiado, se consideran tres actitudes a ser evaluadas: satisfacción, involucramiento y compromiso organizacional ${ }^{(1)}$. De estas tres, la satisfacción laboral es la principal actitud estudiada, seguida por el compromiso con el trabajo; es decir, cuánto una persona se identifica con su empleo y cómo un trabajador se compromete con su organización y con sus metas ${ }^{(1,2)}$. Por otro lado, la satisfacción laboral se define como la actitud general, positiva o negativa que asume una persona frente a su trabajo; está basada en las creencias y valores que desarrolla de su empleo ${ }^{(2)}$.

La Teoría Motivacional o Bifactorial sobre satisfacción en el en el trabajo de Herzberg, afirma que el trabajo puede ser motivador en sí mismo, y generó siete factores motivacionales o de satisfacción: logro, reconocimiento, trabajo en sí, responsabilidad, progreso, éxito y trabajo desafiante. Estos factores tienen relación directa en cómo el trabajador se siente en su empleo, por lo que se denominaban factores intrínsecos ${ }^{(3)}$. Posteriormente, se encontraron factores nuevos para determinar la insatisfacción, denominados extrínsecos, a los cuales Herzberg denominó higiénicos, entre ellos: las políticas, la organización, dirección de la empresa, supervisión, salario, relaciones interpersonales, seguridad y condiciones de trabajo; que no son parte intrínseca del empleo pero se relacionan con las condiciones bajo las que se ejecuta.

La caracterización de estos factores, permite elaborar instrumentos para evaluar la satisfacción laboral entre los trabajadores de una organización, mediante dos tipos de análisis: a) satisfacción general, definida como la actitud que asume el trabajador frente a todas las facetas de su empleo; b) satisfacción frente a aspectos específicos, como: reconocimiento, remuneración, beneficios, condiciones del trabajo, supervisión, compañeros, políticas empresariales, etc ${ }^{(4)}$.

Sin embargo, un grupo laboral con características peculiares constituye el de docentes, y específicamente en docentes universitarios, ya que estudios previos han encontrado altos niveles de satisfacción general, refiriendo dentro de las ventajas la posibilidad de una carrera de promoción docente, la libertad para ejercer la catedra y la relación de este trabajo con la posibilidad de realizar investigación científica; a pesar de que a veces las condiciones de infraestructura y salario no sean las más adecuadas ${ }^{(5,6,7)}$.

En nuestro país, algunos estudios ya han explorado la situación en docentes universitarios; sin embargo, la mayoría de estos estudios se han realizado en universidades privadas, donde los factores extrínsecos de satisfacción suelen ser muy distintos, y en algunos casos, más favorables en comparación a lo que se encuentra en universidades públicas ${ }^{(8-11)}$.

Existen factores adicionales en docentes que ejercen esta actividad en escuelas en las que se forman profesionales de la salud, debido a que estas profesiones suelen presentar altas cargas académicas e implican grandes inversiones de tiempo por parte de los estudiantes y docentes. En la Facultad de Medicina de la Universidad Nacional Mayor de San Marcos, se encuentra el Departamento Académico de Ciencias Morfológicas, que imparte los cursos de anatomía e histología y embriología, los que usualmente son parte de la primera etapa de formación en la facultad. Sus actuales instalaciones se inauguraron en 1887 , por lo que sus ambientes cumplen con pocos criterios de bioseguridad, sobre todo si consideramos que el formaldehido, principal insumo con que se trabaja, es muy toxico ${ }^{(12)}$. Asimismo, el número de alumnos es creciente, y la rotación de docentes nuevos es alta, ya que se producen cambios a otros departamentos.

Dada la importancia del tema y el evidente desconocimiento sobre la satisfacción laboral en el docente del Departamento de Ciencias Morfológicas de la Facultad de Medicina de la Universidad Nacional Mayor de San Marcos, se establece el objetivo de medir el índice de satisfacción laboral en relación al trabajo en general, remuneración, trabajo actual, capacitación, oportunidades, colegas y supervisión, durante el semestre 2014-II.

\section{MÉTODOS}

\section{Diseño del estudio}

Estudio descriptivo, cuantitativo, de corte transversal realizado el semestre 2014-II, que se realizó en docentes nombrados del Departamento Académico de Ciencias Morfológicas de la Facultad de Medicina de la Universidad Nacional Mayor de San Marcos.

\section{Población y muestra}

Se entrevistó a 21 docentes nombrados del Departamento Académico de Ciencias Morfológicas (46,6\% del total de docentes de dicho Departamento), los que respondieron un cuestionario y dieron su autorización para la participación en el estudio. Se utilizó el cuestionario del Índice Descriptivo del Trabajo (JDI) - Bowling Green State University ${ }^{(13,14,15)}$. Previamente se validó el cuestionario aplicándolo en 10 docentes de la Escuela de Nutrición de la misma facultad.

\section{Variables de estudio}

El cuestionario solicita información de algunas características socio-demográficas (edad, sexo, tiempo de servicio), y luego se solicitó a los entrevistados que describan su trabajo considerando cinco dimensiones específicas: trabajo actual (18 ítems), remuneración (9 ítems), oportunidades (9 ítems), supervisión (18 ítems), colegas (18 ítems) y una dimensión general (18 ítems). Al tener las facetas de remuneración y oportunidades, 9 ítems, fue necesario duplicar el valor, sumando en total 108 ítems.

Cada texto del ítem es de fácil comprensión, y en las respuestas se aplicaron según una escala tipo Likert: 'Sí, 'Dudoso' o 'No' con los valores de 3, 1 y 0 respectivamente. Con la suma de puntos se tuvo tres categorías: satisfechos, sentimientos neutrales o ambivalentes e insatisfechos. La suma de los puntos a cada escala refleja un valor del grado de satisfacción, el cual es comparado con la escala establecida para el JDI que va desde 0 hasta 54, este último es el valor máximo posible que se puede obtener al sumar el puntaje dado a cada ítem en cada escala.

Considerando este valor el intermedio es 27, a partir del cual los especialistas 
del JDI señalaron que cinco puntos arriba y cinco puntos abajo de este valor, delimitan una zona llamada de "sentimientos neutrales o ambivalentes", lo cual no refleja satisfacción ni insatisfacción. De igual forma, puntajes mayores de 32 reflejaron satisfacción y puntajes menores de 22 insatisfacción.

\section{Análisis estadístico}

Una vez aplicadas las encuestas, se procedió a la elaboración de una base de datos, para el análisis de la información obtenida. Posteriormente, se realizaron estadísticas descriptivas, frecuencias absolutas y relativas (porcentajes), en el caso de variables cualitativas como el sexo o las categorías de edad y tiempo de servicio; y promedios \pm desviaciones estándar, en variables cuantitativas como las dimensiones del cuestionario JDI y su valoración global.

\section{Aspectos éticos}

El presente estudio fue aprobado por el Comité de Ética de la Facultad de Medicina de la Universidad Nacional Mayor de San Marcos. Las encuestas se realizaron de manera anónima, por lo que no se consignó ningún dato personal que pudiera llevar a la identificación de los encuestados.

\section{RESULTADOS}

La edad promedio de los entrevistados fue 62,3 \pm 7,7 años, los mayores fueron de la Sección Anatomía, con 64,2 \pm 7,4 años; los de la Sección Histología y Embriología tuvieron en promedio de 59,1 \pm 8,1 años. En anatomía, el mayor número de docentes estuvo entre los 50 y 59 años $(28,6 \%)$ y la tendencia fue creciente, encontrándose un $14 \%$ de mayores de 70 años En histología y embriología el rango fue más amplio; así, sus edades fluctuaron entre 50 y 69 años (23,8\%).

El $71,4 \%$ fueron de sexo masculino, el $61,8 \%$ fueron de anatomía. El 61,9\% tuvieron entre 30 a 39 años de servicio, en anatomía el $47,6 \%$, y en histología y embriología el 14,2\%. Adicionalmente, en esta última sección se observó que la mayor cantidad de docentes tuvieron entre 20 y 29 años de servicio (19\%). Sólo en anatomía se observó un profesor con menos de 10 años de servicio (4,8\%) (Tabla 1$)$.
Tabla 1. Características socio-epidemiológicas de los docentes encuestados según secciones del Departamento Académico de Ciencias Morfológicas de la Facultad de Medicina de la Universidad Nacional Mayor de San Marcos, 2014.

\begin{tabular}{|c|c|c|c|c|c|c|}
\hline \multirow[t]{2}{*}{ Características } & \multicolumn{2}{|c|}{$\begin{array}{c}\text { Departamento } \\
\text { Académico de } \\
\text { Ciencias Morfológicas }\end{array}$} & \multicolumn{2}{|c|}{$\begin{array}{l}\text { Sección } \\
\text { Anatomía }\end{array}$} & \multicolumn{2}{|c|}{$\begin{array}{c}\text { Sección Histología y } \\
\text { Embriología }\end{array}$} \\
\hline & $\mathbf{n}$ & $\%$ & $\mathrm{n}$ & $\%$ & $\mathbf{n}$ & $\%$ \\
\hline \multicolumn{7}{|l|}{ Edad (años) } \\
\hline $46-49$ & 1 & 4,8 & 0 & 0 & 1 & 4,8 \\
\hline $50-59$ & 9 & 42,9 & 6 & 28,6 & 3 & 14,3 \\
\hline $60-69$ & 7 & 33,3 & 5 & 23,8 & 2 & 9,5 \\
\hline$>70$ & 4 & 19,0 & 3 & 14,3 & 1 & 4,8 \\
\hline \multicolumn{7}{|l|}{ Sexo } \\
\hline Masculino & 15 & 71,4 & 13 & 61,8 & 2 & 9,5 \\
\hline Femenino & 6 & 28,6 & 1 & 4,8 & 5 & 23,8 \\
\hline \multicolumn{7}{|c|}{ Tiempo de servicios (años) } \\
\hline $0-19$ & 1 & 4,8 & 1 & 4,8 & 0 & 0 \\
\hline $20-29$ & 5 & 23,8 & 1 & 4,8 & 4 & 19,0 \\
\hline $30-39$ & 13 & 61,9 & 10 & 47,6 & 3 & 14,3 \\
\hline$>40$ & 2 & 9,5 & 2 & 9,6 & 0 & 0 \\
\hline
\end{tabular}

En relación al trabajo en general, los docentes mostraron un criterio de indiferencia, con un promedio de $26,7 \pm$ 5 puntos; $25,1 \pm 5,4$ puntos para los de anatomía y $29,7 \pm 2,3$ puntos para los de histología y embriología. Respecto a las dimensiones específicas, el trabajo actual mostró un índice de satisfacción positivo general con una media de 35,1 $\pm 5,9 ; \quad 33,9 \pm 6,9$ en anatomía y 37,6 \pm 2,0 en histología y embriología. Respecto a las variables supervisión, colegas, y oportunidades de progreso, los docentes mostraron indiferencia o neutralidad. La remuneración fue la única variable donde estuvo muy manifiesta la insatisfacción con una media de 17,8 \pm 5,9 puntos; siendo $16,3 \pm 3,4$ puntos para anatomía y 20,9 $\pm 8,6$ puntos para histología y embriología (Tabla 2).

\section{DISCUSIÓN}

Según nuestros hallazgos, y en relación a los factores demográficos, la edad promedio de 62,3 años reveló una marcada diferencia con otras investigaciones donde se reportaron docentes de menor edad $(9,10,11,16)$. El tiempo de servicio de 30 a 39 años (61\%) igualmente mostró diferencias, siendo tiempos mayores al promedio encontrado en otros estudios $(9,16,17)$

El 'trabajo en general' evidenció sentimientos neutrales (26,7 puntos), lo que
Tabla 2. Satisfacción laboral según trabajo en general y dimensiones específicas del Índice Descriptivo del Trabajo (JDI) en docentes del Departamento Académico de Ciencias Morfológicas de la Facultad de Medicina de la Universidad Nacional Mayor de San Marcos, 2014.

\begin{tabular}{lcccccc} 
Satisfacción Laboral & \multicolumn{2}{c}{$\begin{array}{c}\text { Departamento } \\
\text { Académico de } \\
\text { Ciencias Morfológicas }\end{array}$} & Sección Anatomía & $\begin{array}{c}\text { Sección Histología y } \\
\text { Embriología }\end{array}$ \\
\cline { 2 - 7 } Media & D.E. & Media & D.E. & Media & D.E. \\
\hline Trabajo en general & 26,7 & 5,0 & 25,1 & 5,4 & 29,7 & 2,3 \\
\hline Trabajo Actual & 35,1 & 5,9 & 33,9 & 6,9 & 37,6 & 2,0 \\
\hline Remuneración & 17,8 & 5,9 & 16,3 & 3,4 & 20,9 & 8,6 \\
\hline Oportunidad de ascenso & 29,1 & 5,4 & 27,8 & 4,2 & 31,9 & 6,8 \\
\hline Colegas & 22,9 & 4,8 & 23,4 & 5,4 & 22,0 & 3,4 \\
\hline Supervisión & 28,0 & 5,0 & 27,6 & 5,8 & 28,7 & 2,9 \\
\hline
\end{tabular}

D.E. = Desviación estándar 
no es similar a lo encontrado en otros estudios, donde se determinó un importante grado de satisfacción ${ }^{(8-11,18)}$. Esta situación puede explicarse a la innegable influencia de factores extrínsecos por un tiempo prolongado de servicio. Cabe destacar que en este departamento académico se presentaron cambios en la infraestructura de anatomía, no asi en histología, aún cuando las condiciones de trabajo siguen siendo las mismas.

Respecto al 'trabajo actual', los docentes estuvieron satisfechos (35,1 puntos), similar a lo reportado por Carrillo ${ }^{(8)}$, Olivares ${ }^{(9)}$ y Saldaña ${ }^{(18)}$, y que corrobora el hecho de que la docencia es una actividad que genera mucha satisfacción en las personas que la desempeñan. En lo que se refiere a 'oportunidades de progreso', 'relación con colegas' y 'supervisión', los sentimientos fueron neutrales, semejante a lo reportado en otras investigaciones ${ }^{(8,9,11,18)}$, lo que implica que estas dimensiones suelen no ser priorizadas en personas que se dedican a la docencia universitaria.

El aspecto 'remunerativo' presentó una insatisfacción muy marcada $(17,8$ puntos), posiblemente relacionado a que si bien en los últimos años la remuneración de los docentes se incrementó en un $20 \%$ aproximadamente, no creemos que esto promueva un cambio en el sentimiento de los profesores respecto a su honorario. Esta variable ha sido evaluada en estudios realizados en universidades públicas, en los que el sentimiento de insatisfacción se replica, como en las investigaciones de Álvarez ${ }^{(16)}$ y Pronay ${ }^{(6)}$; no así en las universidades privadas, en donde los sentimientos son neutrales ${ }^{(9)} y$ de satisfacción en algunos casos ${ }^{(7,16)}$.
Una de las limitaciones de nuestro estudio fue la muestra pequeña y el análisis en solo uno de los 13 departamentos académicos que tiene la facultad. Si bien el estudio se realizó en el año 2014, consideramos que dadas las mismas condiciones en la labor docente hasta la actualidad, probablemente los hallazgos tengan poca variación.

Concluimos que los docentes de ciencias morfológicas, la mayoría con edad muy avanzada y con mucho tiempo de servicio, mostraron un buen nivel de satisfacción cuando se menciona al trabajo en sí, independiente de la remuneración y las otras variables evaluadas.

\section{AGRADECIMIENTOS}

Al Dr. José Arroyo Acevedo y a la Mg. Acela Arnao Salas por la orientación y asesoría para la presente investigación.

\section{REFERENCIAS BIBLIOGRÁFICAS}

1. Robbins. Comportamiento Organizacional, $15^{\circ}$ Edición. México: Pearson Educacion de Mexico; 2013.

2. Robbins $\mathrm{S}$, Coulter M. Administración $-12^{\mathrm{a}}$ Edición México: Pearson Educación; 2014.

3. Herzberg F, Mausner B, Snyderman BB. The Motivation to Work. Transaction Publishers; 1959.

4. Pérez J, Fidalgo M. Satisfacción laboral: escala general de satisfacción. Nota técnica de prevención 394 [On line]. Madrid: Instituto de Seguridad e Higiene en el Trabajo. Ministerio de Trabajo y Asuntos Sociales; 1995 [citado 31 marzo 2019]. Disponible en: http://www.insht.es/InshtWeb/Contenidos/Documentacion/FichasTecnicas/NTP/Ficheros/301a400/ ntp 394.pdf

5. Rodríguez D, Núñez L, Renere AC. Estudio comparativo de la satisfacción laboral universitaria en el núcleo de Anzoátegui de la Universidad de Oriente. Investig Postgrado. 2010;25(1):63-80
6. Pronay B. Job Satisfaction of Non-government College Teachers in Bangladesh. JEduc Pract. 2011;2(4):87-91-91.

7. Saba I. Measuring the Job Satisfaction Level of the Academic Staffin Bahawalpur Colleges. Int J Acad Res Bus Soc Sci. 2011;1(1):12-9.

8. Carrillo SP. Motivación y clima laboral en personal de entidades universitarias. Rev Investig En Psicol. 2000;3(1):11-21. DOI: 10.15381/rinvp.v3i1.4909

9. Olivares Preciado JA, Quintana Del Solar MG, Matta Morales CO, Choy Lisung J, Ronquillo Herrera WJ, De las Mercedes M, et al. Satisfacción laboral de docentes universitarios del Departamento Académico de Clínica Estomatológica. Rev Estomatol Hered. 2006;16(1):21-5.

10. Alfaro Peña G. Satisfacción laboral de los docente en la Universidad Científica del Sur, Villa el Salvador, 2015 [Tesis de Magister]. Lima: Universidad César Vallejo; 2015 [citado 12 enero 2019]. Disponible en: http://repositorio. ucv.edu.pe/handle/UCV/5076

11. Regalado Sanchez S. Presion laboral y satisfaccion laboral en docentes de una universidad privada de trujillo [Tesis para obtener el Título Profesional en Psicologia]. Trujillo: Universidad Privada Antenor Orrego; 2017 [citado 12 enero 2019]. Disponible en: http://repositorio.upao. edu.pe/handle/upaorep/2457

12. Cruz V. La enseñanza de la anatomía en el Perú-El anfiteatro anatómico. Bol San Fernandino Fac Med UNMSM. 2000;8(4):38-61.

13. Kinicki AJ, Mckee-Ryan FM, Schriesheim CA, Carson KP. Assessing the construct validity of the job descriptive index: a review and meta-analysis. J Appl Psychol. 2002;87(1):14-32. DOI: 10.1037/0021-9010.87.1.14

14. Smith PC, Kendall LM, Hulin CL. The measurement of satisfaction in work and retirement: A strategy for the study of attitudes. Oxford, England: Rand Mcnally; 1969.

15. Brodke M, Sliter M, BalzerW, Gillespie J, Gillespie M. The Job Descriptive Index and Job in General (2009 - Revision) Quick Reference Guide. Department of Psychology. Bowling Green State University.; 2009.

16. Alvarez Flores D. Fuentes de presión laboral y satisfacción laboral en docentes de universidades estatales y universidades privadas de Lima Metropolitana [Tesis Doctoral On line]. Lima: Universidad Nacional Mayor de San Marcos; 2007 [citado12 décembre 2019]. Disponible en: http:// cybertesis.unmsm.edu.pe/handle/cybertesis/560

17. Pujol-Cols LJ. Satisfacción Laboral en docentes universitarios: medición y estudio de variables influyentes. REDU Rev Docencia Univ. 2016;14(2):261-92. DOI: 10.4995/ redu.2016.5974

18. Saldaña Labajos A. Clima laboral y satisfacción laboral en el profesional de salud de emergencia del Hospital Nacional Sergio Enrique Bernales Lima 2016 [Tesis de Magister]. Lima: Universidad César Vallejo; 2017 [citado 12 enero 2019]. Disponible en: http://repositorio.ucv.edu.pe/handle/UCV/8718 